\title{
OFDM Transmission Technique to Minimize PAPR
}

\author{
Ahmad Fadzil Ismail ${ }^{1}$, Sofia Pinardi ${ }^{1}$, Mohammad Kamrul Hassan ${ }^{1}$, R.M.A Raj \\ Mohamed $^{2}$ and Wahidah Hashim ${ }^{2}$ \\ ${ }^{1}$ Department of Electrical and Computer Engineering, \\ International Islamic University Malaysia, \\ ${ }^{2}$ College of Computer Science and Information Technology, \\ Universiti Tenaga Nasional Malaysia \\ sofiapinardi@yahoo.com,af_ismail@iium.edu.my,hasankamrul@ieee.org, \\ Wahidah@uniten.edu.my
}

\begin{abstract}
Multi-carrier phenomenon Orthogonal Frequency Division Multiplexing (OFDM) is considered to be one of the most significant developments in wireless communication. It is now becoming somewhat a critical important standard. However, high Peak Average Power Ratio (PAPR) is a key issue in OFDM systems. It resulted the lowering of power efficiency. Conventionally OFDM can be implemented using Discrete Fourier Transform (DFT) technique that usually adopts modulation either Binary Phase Shift Keying (BPSK), Quadrature Phase Shift Keying (QPSK) or Quadrature Amplitude Modulation (QAM). In the study, OFDM systems employing DFT, Discrete Wavelet Transform (DWT) and Discrete Cosine Transform (DCT) had been investigated by means of computer simulation. The main aim is to assess the enactment of the Bit Error Rate (BER) and PAPR in OFDM system. The performance of the system was assessed in the occurrence of Additive white Gaussian noise (AWGN) as well as incorporating multipath fading as the channel impairments. The acquired preliminary findings show that the DWT technique outperforms DFT and DCT. It is therefore deemed that DWT based OFDM has the potential to significantly reduce PAPR.
\end{abstract}

Keywords: OFDM, PAPR, BER, DFT, DWT, DCT

\section{Introduction}

With the rapid demands of mobile communication services and broadband mobile internet access services, improving the capability of the wireless communication system is a requirement. At present, in addition to voice services, up to about five megabit rate data services are already made available to the fourth generation $4 \mathrm{G}$ mobile users in select parts of the world. However, the demands for wireless broadband multimedia communication system are anticipated to require much higher transfer rate in the hope to be able to accommodate both the public and private sector. In order to create a free flowing broadband multimedia mobile communication system, it is therefore necessary to achieve higher bit rate and enhanced throughput. The fifth generation mobile systems commonly abbreviated as 5G, are currently being assembled in the hope to accomplish faster connection speeds than the 4G.There is this catch though in the race toward the greater bandwidth. On the off chance that the advanced information is being transmitted at the rate of a few megabits for every second, the defer time of the postpone waves is to be sure more noteworthy than 1 symbol time [1].

The general consensus is that the $5 \mathrm{G}$ will adopt OFDM as its multicarrier modulation scheme. OFDM is a scheme that is regarded as having the capability to accomplish high performance over multipath environments and at present is already adopted in many 
wireless applications. The data are transmitted using numerous narrow-band orthogonal sub-carriers when OFDM is engaged[1]. It is also envisioned that the dominant air interface for the $5 \mathrm{G}$ broadband wireless communications will also incorporate multiple input multiple output (MIMO) technology [2]. Such MIMO-OFDM setup is said to be a promising technique capable of enhancing the channel capacity whilst retaining the system robustness against severe multipath fading. [3]. OFDM implementation is not without challenges. It encounters high envelope changes and high PAPR, which in the long run prompts to issue with enhancement since direct speakers with high back-off will be required. This from this time forward lessens the intensification effectiveness [4]. Radio recurrence RF speakers are required to be worked in a vast direct locale. This is an exertion made to maintain a strategic distance from the flag crests from abiding in the non-straight area of the power enhancer which eventually cause flag bending. With flag mutilation, intermodulation's among the subcarriers and out of band radiation is presented. These are the justifications why the power amplifiers should be at all-time be driven with large power back-offs. Then again, large power back-offs lead to a very inefficient amplification and requires expensive transmitters. Therefore the PAPR reduction is a critical design aspect for the so-call to-be-invented MIMO-OFDM system.

\section{OFDM Transform Techniques}

The OFDM transformation techniques are discussed as below:

\subsection{Discrete Cosine Transform}

DFT and inverse DFT (IDFT) procedures can send inside the usage of OFDM transmission framework [7]. The OFDM conspire likewise embeds a monitor interim at time space also can mention as the cyclic prefix $(\mathrm{CP})$, which alleviates the ISI among OFDM symbols. In Figure 1 OFDM framework in AWGN, Rayleigh blurring channel and recreation is shown. Plainly BER execution in an AWGN channel is reliable with the logical outcomes. The discrete cosine transform (DCT) isolates the picture into parts (or unearthly sub-groups) of contrasting significance (concerning the picture's visual quality). The DCT is like the discrete Fourier change; it changes a flag or symbol from the spatial area to the frequency. The expository BER expressions for M-array QAM motioning in AWGN and Rayleigh directs are individually spoken to in Eqn.1. furthermore, in Eqn.2. [7].

$$
G_{x}(0)=\frac{\sqrt{2}}{M} \sum_{m=0}^{M-1} X(m)
$$

where $G_{x}(k)$ is the $k^{\text {th }}$ DCT factor, it is worthwhile nothing that the usual basis vectors $\{1 / \sqrt{2}, \cos ((2 \mathrm{~m}+1) \mathrm{k} \pi) /(2 \mathrm{M})\}$ is actually a class of discrete chebyshev polynomials. Inverse cosine discrete transform (ICDT) is defined as:

$$
x(m)=\frac{1}{\sqrt{2}} G_{x}(0)+\sum_{k=1}^{M-1} G_{x}(k) \cos \frac{(2 m+1) k \pi}{2 M}
$$

Where $\gamma$ and $M$ denote $E_{b} / N_{0}$ as well as modulation direction, Q(.) states the Q-function which is expressed in Eqn.3.

$$
Q(x)=\frac{1}{\sqrt{2 \pi}} \int_{x}^{\infty} e^{-t^{2} / 2} d t
$$




\subsection{Discrete Wavelet Transform (DWT)}

The DWT resembles the Fourier change with an absolutely exceptional authenticity work. The essential differentiation is this, Fourier change separates the banner into sines and cosines, i.e. the limits restricted in Fourier space; in inverse, the wavelet change uses limits that are bound as a piece of both the bona fide and Fourier space. All around, the wavelet change can be imparted by the going with condition:

$$
F(a, b)=\int_{-\infty}^{\infty} f(x) \psi_{(a, b)}^{*}(x) d x
$$

where the $*$ is the complex conjugate symbol and $\psi$ is a function which can be picked subjectively given that it complies with specific standards. The DWT is a usage of the wavelet transform utilizing a discrete arrangement of the wavelet scales and interpretations complying with some characterized rules. At the end of the day, this change deteriorates the flag into a commonly orthogonal arrangement of wavelets, which is the primary contrast from the ceaseless wavelet change (CWT), or its usage for the discrete time arrangement now and then called DT-CWT. The arrangement of scaled as well as moving wavelets organizes an orthogonal premise of DWT, showing a pertinent trademark put resources into the subcarriers covering [6]:

$$
\psi_{(m, n)}(t)=2^{\frac{m}{2}} \psi\left(2^{m}(t)-n\right)
$$

where $\psi(t)$ indicates the wavelet, $m$ and $n$ are the scale and decipher parameters individually. It is characterized the capacity $\phi(t)$ is the scaling capacity or the main wavelet which moves and scales. $\psi(t)$ and $\phi(t)$ are utilized $\phi(t)$ together to prepare any flag and examine it.

$$
\begin{gathered}
\emptyset(t)=\sum_{n} h_{\emptyset} \sqrt{2 \emptyset(2 t-n)} \\
\psi(t)=\sum_{n} h_{\psi} \sqrt{2 \psi(2 t-n)}
\end{gathered}
$$

$h(n)$ and $h \psi(n)$ are individually a low pass and a high pass channel banks. The low pass channel gives rough data about the flag while the high-pass channel produces itemized information condition:

\subsection{Fourier Based-OFDM}

OFDM is another kind of multichannel framework. The various orthogonal subcarrier signals, which are covered in the range, can be created by summing up the single transporter into the multicarrier signals. DFT and IDFT procedures can valuable for executing in the OFDM transmission framework [7]. The OFDM conspire additionally embeds a monitor interim in the time area, called cyclic prefix $(\mathrm{CP})$, which mitigates the ISI between OFDM images. Figure 1 demonstrates the OFDM framework in AWGN, Rayleigh blurring channel and recreation. Obviously BER execution in an AWGN channel is predictable with the logical outcomes.

\section{A. BER of OFDM scheme}

The analytical BER expressions for M-array QAM signaling in AWGN (in Eqn.8)and Rayleigh channels(in Eqn. 9) are respectively given as [2]:

$$
\begin{gathered}
P_{e}=\frac{2(M-1)}{M \log _{2} M} Q\left(\sqrt{\frac{6 E_{b}}{n_{0}} \cdot \frac{\log _{2} M}{M^{2}-1}}\right) \\
P_{e}=\frac{M-1}{M \log _{2} M}\left(1-\sqrt{\frac{3 \gamma \log _{2} M /\left(M^{2}-1\right)}{3 \gamma \log _{2} M /\left(M^{2}-1\right)+1}}\right)
\end{gathered}
$$


Where $\gamma$ and $M$ denote $E_{b} / N_{0}$ standard Q-function defined as:

and modulation order, respectively, while $Q($.$) is the$

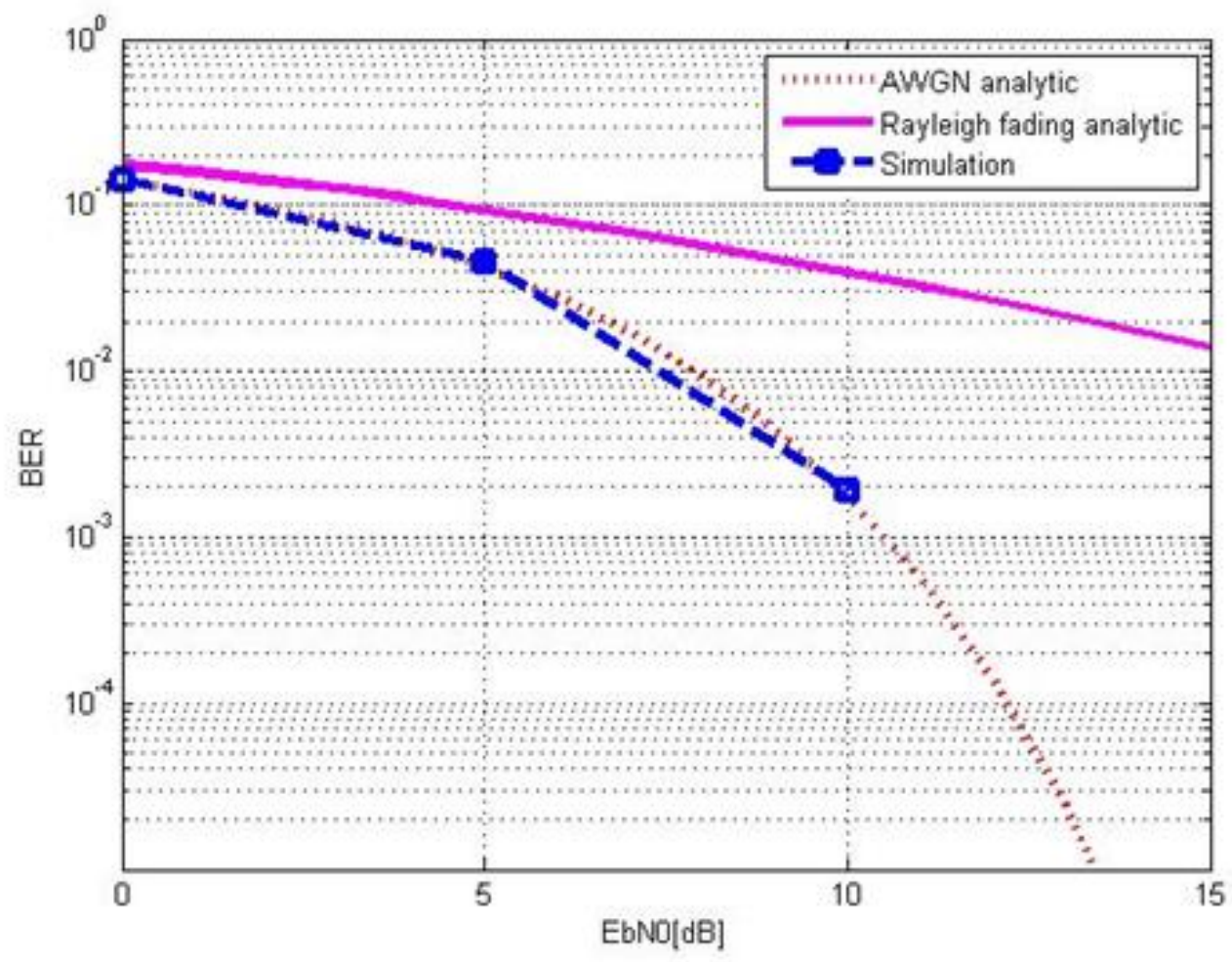

Figure 1. The OFDM System in AWGN and Rayleigh Fading Channel

\subsection{Wavelet Based-OFDM Transformation}

WT is a multiplexing transmission strategy in which data is distributed to sub-bunches with different time and repeat resolutions. While Fourier examination which isolates the whole transmission limit into orthogonal and covering sub-gatherings of identical information transmissions. This allows a kind of time-frequency examination (or elucidation scale in wavelet talks). Wavelet highlight can give much lower side folds degree than those of FT and the loss of orthogonality prompts to lesser ISI and ICI. Along these lines, OFDM requires time and repeat synchronization to get a tolerable execution. This can be overcome by the use of the WT-based OFDM approach. The FFT impede in the primary diagram is as of now supplanted by the DWT square. The pictures came to fruition on account of star gathering mapping background IDWT operations and construct the looked for OFDM hail. The operation is done by the guide of quadrature mirror channels that are the low pass and high pass IDWT channel banks. Come full circle entertainment and orthonormal start properties are satisfied, where pictures are convoluted with those channels and after that multiplexed together forming the banner. 


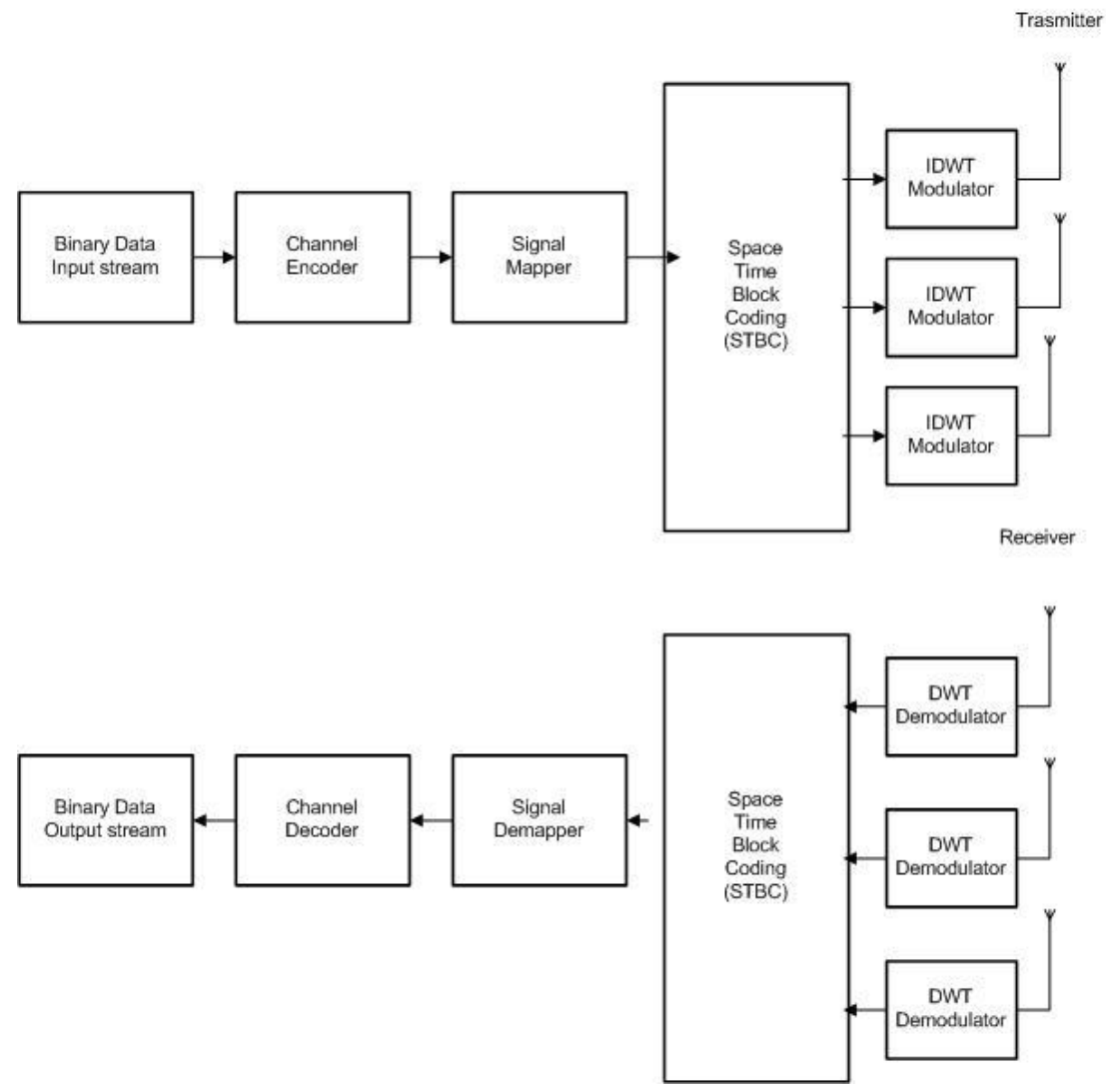

Figure 2. Block Diagram of DWT based OFDM Transceiver

The multicarrier handset DWT based OFDM is showed up in Figure 2 . The FFT in OFDM is supplanted by Inverse DWT as modulator at the transmitter furthermore DWT as demodulator at the recipient [8] . The routine OFDM, and DWT multicarrier plan, is the clearing of the cyclic prefix impedes in the handset. The families wavelet mothers to be particular " Haar'.

\subsection{Peak Average Power Ratio (PAPR)}

The transmit signal in an OFDM framework ha high pick values in the time area since numerous subcarrier parts are included by means of an IFFT operation. Hence, OFDM frameworks are known to have a high Peak Average Power Ratio (PAPR), contrasted and single transporter framework. The effect of high PAPR diminishes the Signal to Noise Ratio (SNR) of Analog to Digital Converter (ADC) and Digital to Analog Converter (DAC) while debasing the proficiency of the power intensifier in the transmitter. The PAPR is more vital in the uplink since the effectiveness control speaker is basic because of the restricted battery control in a versatile terminal. For the OFDM flag, PAPR is characterized as the proportion of maximal flag esteem and its normal power $[7,9]$ :

$$
\operatorname{PAPR}(x(t))=\frac{\max \left(|x(t)|^{2}\right)}{E\left\{|x(t)|^{2}\right\}}
$$

Where $E($.$) denotes the expected value, where \mathrm{N}=$ amount of subcarrier. In the case of QPSK modulation, each subcarrier has the constant amplitude $\left|A_{k}\right|=1$ and the maximal theoretical PAPR value in digital domain can be calculated as:

$$
P P A P R=10 \log 10(N)
$$




\section{Result and Discussion}

The simulation is carried out using Matlab tool. The system parameter for the simulation is given in Table 1 which are used to comparing DFT, DCT, and DWT for minimizing PAPR.

Table 1.System Parameter

\begin{tabular}{|c|c|c|c|}
\hline Variable & DFT-OFDM & DCT-OFDM & DWT-OFDM \\
\hline $\begin{array}{c}\text { Number of } \\
\text { sub-carrier }\end{array}$ & 64 & 64 & 64 \\
\hline Message size & 10 & 10 & 10 \\
\hline $\begin{array}{c}\text { Number of } \\
\text { cyclic prefixes }\end{array}$ & 4 & 4 & - \\
\hline Wavelet used & - & - & Haar \\
\hline $\begin{array}{c}\text { Modulation } \\
\text { PSK }\end{array}$ & Binary & Binary & Binary \\
\hline
\end{tabular}

Hypothetically, wavelet change guarantees an enhanced execution in correlation with Fourier and cosine change. The Matlab reenactments were done to accomplish this objective. In Figure 2 and Table 2, demonstrate that BER execution for DWT-OFDM framework superior to DFT-OFDM and DCT-OFDM. Reproduction is done for SNR in the range 1 to $7 \mathrm{~dB}$, for BPSK as an adjustment system; the BER of DFT-OFDM and DCT-OFDM are under $10^{-2}$ for SNR in $3 \mathrm{~dB}$, while the BER of DWT-OFDM has achieved $10^{-3}$. In recurrence specific blurring, BER execution of DWT-OFDM is over 100 for SNR in $20 \mathrm{~dB}$ which can be found in Figure 3. The PAPR values for BPSK regulation are accounted for in Figure 4 and Table 3.

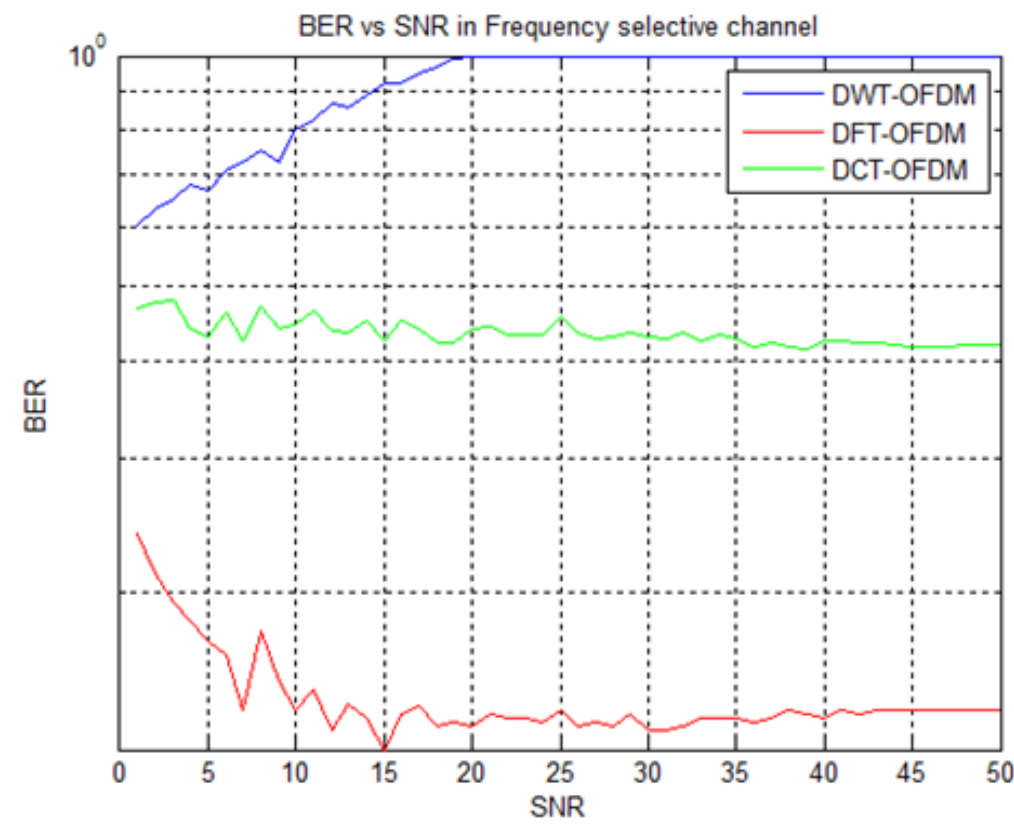

Figure 2. BER performance of DFT-OFDM, DCT-OFDM, and DWT-OFDM in AWGN Channel 


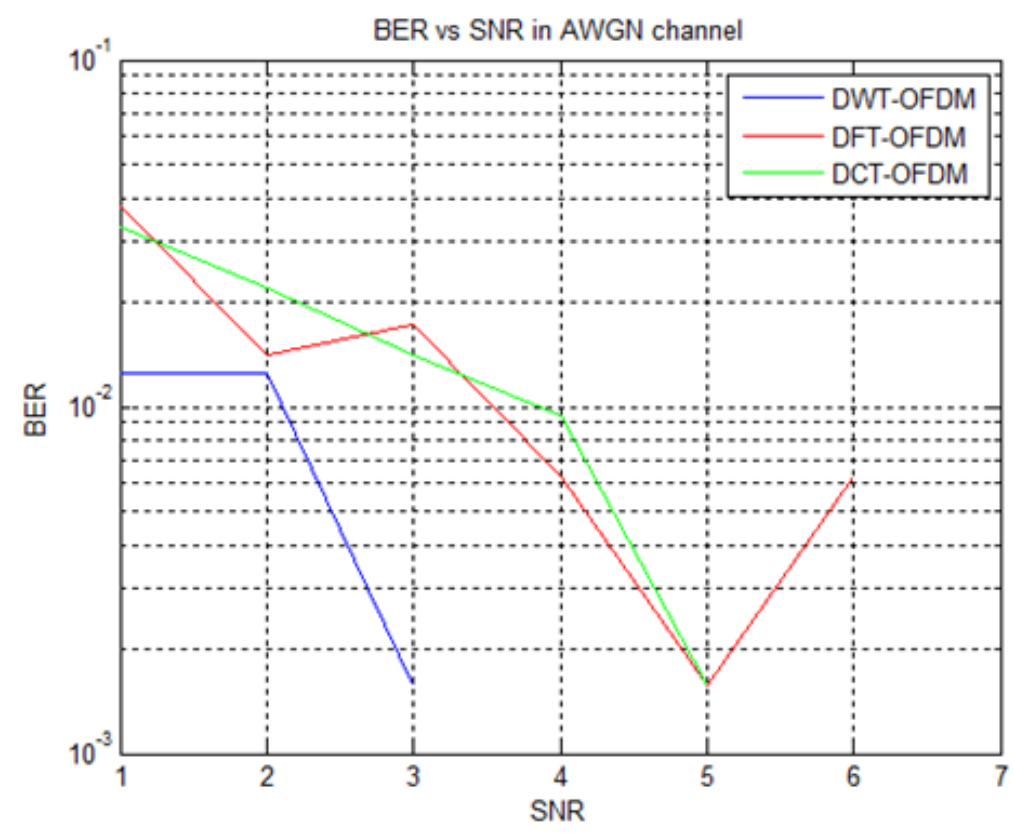

Figure 3. BER Performance of DFT-OFDM, DCT-OFDM, and DWT-OFDM in Frequency Selective Channel

Table 2. BER Performance for DFT,DCT, and DWT-OFDM

\begin{tabular}{|c|c|c|}
\hline Techniques & SNR(dB) & BER \\
\hline DFT & 3 & $10^{-2}$ \\
\hline DCT & 3 & $10^{-2}$ \\
\hline DWT & 3 & $10^{-3}$ \\
\hline
\end{tabular}

The PAPR values for a number of carrier 64 in DWT-OFDM reach $4 \mathrm{~dB}$, it is better than DFTOFDM $(19 \mathrm{~dB})$ and DCT-OFDM $(18 \mathrm{~dB})$. It is important to note that even the value 18 or $19 \mathrm{~dB}$ is considered as high PAPR because it implies that the peak value is more than one order of magnitude stronger than the average signal value.

Table 3. PAPR Comparison for DFT, DCT, and DWT-OFDM

\begin{tabular}{|c|c|}
\hline No. of carrier & $\mathbf{6 4}$ \\
\hline PAPR for DFT-OFDM $(\mathrm{dB})$ & 19 \\
\hline PAPR for DCT-OFDM $(\mathrm{dB})$ & 18 \\
\hline PAPR for DWT-OFDM $(\mathrm{dB})$ & 4 \\
\hline
\end{tabular}




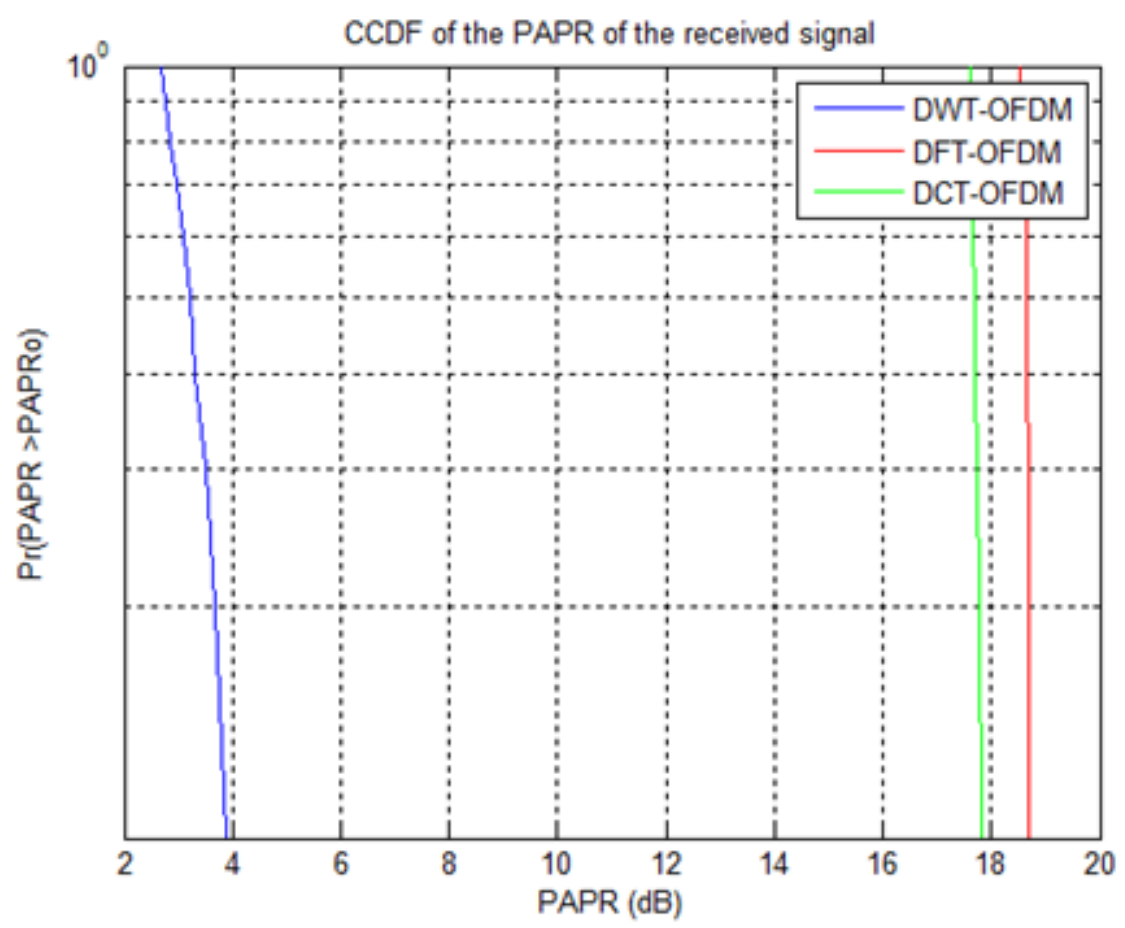

Figure 4. Influence a Function of Time and Frequencies (DFT,DCT, and DWTOFDM) to PAPR Value

\section{Conclusion}

This paper studied and analyzed the OFDM transformation techniques in AWGN and Reyleigh fading channel conditions. The simulation has done in order to compare performance of the OFDM transformation techniques. Simulation results show that DWT based OFDM scheme yields the lowest bit error rate for AWGN channel. Likewise, the PAPR in DWT-OFDM is better than DCT and DFT-OFDM. Finally, discrete wavelet transform can be one of technique to reduce PAPR .It is mostly appropriate for the OFDM system when the subcarriers are 128 . The future recommendation will be to reduce the PAPR reduction proposal using wavelet based and MIMO for bias less transmission and identifying the best alternatives in terms of performance enhancement. Afterward an empirical study will be done using USRP and GNU radio.

\section{References}

[1] M. K. Hasan, A. F. Ismail, S. Islam, W. Hashim, and B. Pandey, "Dynamic Spectrum Allocation Scheme for Heterogeneous Network," Wireless Personal Communications, pp. 1-17, 2016.

[2] P. Mukunthan and P. Dananjayan, "Modified PTS with FECs for PAPR Reduction in MIMO-OFDM system with different subcarriers," in 2011 International Symposium on Humanities, Science and Engineering Research, 2011, pp. 89-94.

[3] K. Udagawa, T. Yamaguchi, and F. Maehara, "Inter-symbol Interference Suppression Scheme Using Periodic Signals for Coded MIMO-OFDM Systems," in ICOF 2016; 19th International Conference on OFDM and Frequency Domain Techniques; Proceedings of, 2016, pp. 1-5.

[4] M. Beko, M. Marikj, R. Dinis, and M. Tuba, "Peak-to-average power ratio reduction in multiple-input multiple-output orthogonal frequency-division multiple access systems using geodesic descent method," IET Communications, vol. 10, pp. 212-218, 2016.

[5] N. Ahmed, T. Natarajan, and K. R. Rao, "Discrete cosine transform," IEEE transactions on Computers, vol. 100 , pp. 90-93, 1974.

[6] A. Ghaith, R. Hatoum, H. Mrad, and A. Alaeddine, "Performance Analysis of the Wavelet-OFDM new scheme in AWGN channel," in Communications and Information Technology (ICCIT), 2013 Third International Conference on, 2013, pp. 225-229. 
[7] Y. S. Cho, J. Kim, W. Y. Yang, and C. G. Kang, MIMO-OFDM wireless communications with MATLAB: John Wiley \& Sons, 2010.

[8] D. Meenakshi, S. Prabha, and N. Raajan, "Compare the performance analysis for FFT based MIMOOFDM with DWT based MIMO-OFDM," in Emerging Trends in Computing, Communication and Nanotechnology (ICE-CCN), 2013 International Conference on, 2013, pp. 441-445.

[9] I. Orović, N. Žarić, S. Stanković, I. Radusinović, and Z. Veljović, "Analysis of power consumption in OFDM systems," in MIPRO, 2011 Proceedings of the 34th International Convention, 2011, pp. 653657.

[10] Abhishek Katariya, Amita Yadav, Neha Jain, and Geetam Tomar, "BER Comparison of RS and BCH Coded OFDM Transmission Over Noisy Channel", ”, IEEE International Conference on Computational Intelligence and Communication Networks CICN2011, pp 233- Oct 2011 
International Journal of Hybrid Information Technology

Vol. 10, No.3 (2017) 